\title{
Recovery And The Use Of Postoperative Physical Therapy After Total Hip Or Knee Replacement
}

\author{
L. Groot \\ Erasmus MC, University Medical Center \\ D.A.J.M. Latijnhouwers \\ Leiden University Medical Center \\ M. Reijman \\ Erasmus MC, University Medical Center \\ S.H.M. Verdegaal \\ Alrijne Hospital, Department of Orthopedics \\ T.P.M. Vliet Vlieland \\ Leiden University Medical Center \\ M.G.J. Gademan ( $\nabla$ m.g.j.gademan@lumc.nl) \\ Leiden University Medical Center
}

\section{Research Article}

Keywords: physical therapy, recovery, hip, knee, arthroplasty, osteoarthritis

Posted Date: January 11th, 2022

DOI: https://doi.org/10.21203/rs.3.rs-1187618/v1

License: (c) (i) This work is licensed under a Creative Commons Attribution 4.0 International License. Read Full License 


\section{Abstract}

Background: To investigate the relation between recovery and postoperative physical therapy (PT) usage, including the presence of comorbidities, 6 months after total hip or knee arthroplasties (THA/TKA).

Methods: Multicenter, observational study in primary THA/TKA patients who completed preoperative and 6 months postoperative assessments. The assessments included questions on PT use (yes/no and duration; long term use defined as $\geq 12$ weeks), comorbidities (musculoskeletal, non-musculoskeletal, sensory comorbidities). Recovery was assessed with the HOOS/KOOS on all 5 subdomains. Logistic regression with long term PT as outcome was performed adjusted for confounding including an interaction term (comorbidity*HOOS/KOOS-subdomain).

Results: In total, 1289 THA and 1333 TKA patients were included, of whom 95\% received postoperative PT, $56 \%$ and $67 \%$ received postoperative PT $\geq 12$ weeks respectively. In both THA and TKA group, less improvement on all HOOS/KOOS domain scores was associated with $\geq 12$ weeks of postoperative PT (range Odds Ratios 0.97-0.99). In the THA group the impact of recovery was smaller in patient with comorbidities as non- musculoskeletal comorbidities modified all associations between recovery and postoperative PT duration (Odds Ratios range 1.01-1.05). Musculoskeletal comorbidities modified the associations between Function-in-Daily-Living-and Sport-and-recreation recovery and postoperative PT. Sensory comorbidities only had an effect on Sport-and-recreation recovery and postoperative PT. In the TKA group comorbidity did not modify the associations.

Conclusion: Worse recovery was associated with longer duration of postoperative PT suggesting that PT provision is in line with patients' needs. The impact of physical recovery on the use of long-term postoperative PT was smaller in THA patients with comorbidities.

Trial registration: Registered in the Dutch Trial Registry on March 13, 2012. TRIAL ID NTR3348; registration number: P12.047. https://www.trialregister.nl/trial/3197.

\section{Introduction}

Total hip or knee arthroplasties (THA/TKA) are effective procedures in patients with end stage hip or knee osteoarthritis (OA)(1,2). Overall, THA and TKA show favorable long-term effects, yet the recovery process may take weeks to months. Physical therapy (PT) following discharge from hospital is an effective intervention to enhance this recovery process(3-6). Several guidelines recommend the use of PT to improve recovery after THA/TKA(7-9). In Western Europe, post discharge PT after THA/TKA range from 35-99\%(10-12). A rise in use of postoperative PT is expected due to the foreseen increase in arthroplasty procedures $(2,13,14)$.

Previous studies report variation in treatment modalities, the timing and/or the frequency and duration of post discharge PT indicating no clear consensus on its optimal dosage and timing $(10,15-19)$. Also, studies identified determinants that affected use of inpatient versus outpatient PT treatment and its program intensity. It was found that patients referred to the less intensive outpatient PT were more often younger, had less comorbidities, a public insurance status and less functional disability, also referral depended on the treating hospital(10,19-21) as compared to those having more intensive and/or inpatient PT. Understanding the relationships between these factors and the use of postoperative PT could provide better patient expectations, more patient specific advice and targeted postoperative PT care for patients after THA/TKA(22-25).

The patient's physical recovery after THA/TKA, including the presence of comorbidities and its effect on the duration of postoperative PT, has not yet been extensively studied, while it is likely that patients with worse overall health status might have a greater need for PT. To our knowledge only the study of Smith et al., relying on data from the National Joint Registry for England, showed that worse functional outcomes at 12 months after THA/TKA were related to longer duration of PT. However, this relationship was not adjusted for patient characteristics(12).

Therefore, the aim of the present study was to examine the association between recovery and the duration of postoperative PT, taking into account individual patient characteristics and in particular the presence of comorbidities, as these can have a significant influence on the recovery after arthroplasty as well as PT $(26,27)$. A better understanding of the relationships between these factors and the use of postoperative PT could provide new insights into the process of recovery after THA/TKA and targets for optimization of care.

\section{Methods \\ Study design}

This study was part of the ongoing multi-center, prospective cohort Longitudinal Leiden Orthopaedics Outcomes of Osteo-Arthritis study (LOAS) (Trial ID NTR3348)(28). Ethical approval was obtained from the Medical Ethics Committee of the Leiden University Medical Center (Registration number P12.047). The study was performed in accordance with the relevant guidelines and regulations. Since 2012 the LOAS includes consecutive patients scheduled for primary THA/TKA as a result of OA in seven participating hospitals: Leiden University Medical Center, Leiden; Alrijne Hospital, Leiden and Leiderdorp; Groene Hart Hospital, Gouda; Reinier de Graaf Hospital, Delft; Lange Land Hospital, Zoetermeer; Albert Schweitzer Hospital, Dordrecht; and Waterland Hospital, Purmerend. All patients provided informed consent.

\section{Study population}

Patients eligible for the LOAS were aged 18 years or older, physically and/or mentally able to complete questionnaires in Dutch and underwent primary THA/TKA for OA. The present study included a subset of patients from the LOAS who were included between June 2012 and June 2018 , who filled out 
questions on the use of PT pre- and postoperatively and completed the questionnaires on hip/knee function and quality of life both preoperatively and 6 months after surgery.

\section{Assessments}

\section{Patient Characteristics}

Patient characteristics were recorded preoperatively and included age (years), sex, living status (living alone / living with partner, children or other(s)), working status (having a paid job yes/no) and the Body Mass Index (BMI (kg/m2)).

In addition, information on self-reported pain, quality of life and comorbidities were gathered preoperatively. Hip and knee pain severity in rest and during activities in the past week were assessed by the Numeric Rating Scale (NRS)(29), with scores ranging between 0 (no pain) and 10 (worst pain imaginable). Regarding quality of life, the SF-12 was administered (30) from which the Physical and Mental Component Summary scales (PCS and MCS) were computed, with scores ranging between 0 (worst physical/mental health) and 100 (best physical/mental health). Self-reported comorbidities were gathered with a questionnaire developed by the Dutch Central Bureau of Statistics (CBS) (31, 32), in which the presence of comorbidities in the previous year was determined (yes/no). According to the paper of Peter et. al.(27), comorbidities were classified into three domains: Musculoskeletal comorbidities (elbow, wrist, hand, or back pain; other rheumatic diseases), non-musculoskeletal comorbidities (chronic lung, cardiac, or coronary disease; arteriosclerosis; hypertension; [consequences of] stroke; severe bowel disorder; diabetes mellitus; migraine; psoriasis; chronic eczema; cancer; urine incontinence) and sensory impairments (hearing or vision impairments; dizziness in combination with falling). Also the numbers of comorbidities were categorized into 4 groups: none comorbidities, 1-2 comorbidities, $3-4$ comorbidities, and $\geq 5$ comorbidities.

\section{Physical therapy use}

The use of preoperative PT was measured by one question: "Did you have contact with a PT before surgery for your hip or knee complaints in the past 6 months?" (yes/no). The use of postoperative PT was measured by three questions: "Did you receive physical therapy after surgery?" (yes/no); "What was the estimated duration of physical therapy?" (4 weeks, 6 weeks, 8 weeks or 12 weeks or more); and "What was the average frequency of physical therapy?" (Once a week, twice a week or three times per week or more).

\section{Hip and knee related health status recovery and quality of life}

The Hip disability and Osteoarthritis Outcome Score (HOOS) and the Knee injury and Osteoarthritis Outcome Score (KOOS) were used to assess hip and knee related symptoms (health status) preoperatively and 6 months after surgery $(33,34)$. The questionnaires contain 40 and 42 items, respectively, categorized into five domains (function in daily living; pain; symptoms; sport and recreation; quality of life). All scores ranging from 0 (severe impairments) to 100 (no impairments). To determine the impact of recovery on the use of postoperative PT, all domains of the HOOS/KOOS were included. The extent of recovery was expressed as the absolute HOOS/KOOS domain scores at 6 months adjusted for the preoperative values. The distribution based Minimal Clinical Important Differences (MCID) for the HOOS/KOOS domains have been reported to be around 8-9 points(35).

\section{Statistical analysis}

All analyses were performed for THA and TKA separately. Descriptive statistics (mean and standard deviation (SD), median with interquartile range or numbers and percentages) were used to present the patients' preoperative characteristics, level of overall health status, pain and use of PT.

Baseline characteristics were presented according to postoperative PT (divided into none, $<12$ weeks or $\geq 12$ weeks), using One-way ANOVA, Student $t$ tests, Mann-Whitney-U tests or Chi Square tests to test differences between these groups or combinations of these groups, where appropriate patients who did not make use of post-discharge PT were excluded from further analyses. To study the association between recovery and the duration of postoperative PT, the 6month HOOS/KOOS separate domain absolute scores, as well as their change scores were presented for the total group of postoperative PT users and compared between the groups with short and long duration of postoperative PT by means of Paired t tests (results expressed as mean difference in change with the $95 \%$ confidence interval $(\mathrm{Cl})$ ). In addition, to account for potential confounders, logistic regression analyses were performed, in which duration of postoperative PT (< or $\geq 12$ weeks) was the dependent variable and the change scores of the HOOS/KOOS domain scores were each separately considered as independent variables. Comorbidities were included as possible effect modifier and the following potential confounders, which were based on previous literature: age, sex, BMI, living status and mental health(22). For each of the three different comorbidity domain groups (musculoskeletal, non- musculoskeletal and sensory impairments), we performed separate analyses as patients could have several comorbidities and therefore could be included in more than one comorbidity domain. Relations between independent variables were checked for non-linearity. All statistical analyses were performed on complete cases, twosided with 95\% Confidence Intervals (95\% Cl), using SPSS software (IBM Corp. Released 2016. IBM SPSS Statistics for Windows, Version 25.0. Armonk, NY: IBM Corp.).

\section{Results}

\section{Study population}

The flow of participants in this study is presented in FIGURE 1. In total, 2279 and 2164 patients received a primary THA/TKA, respectively. Of these patients, 1289 THA and 1333 TKA patients were finally included for the present analysis. The included THA/TKA patients were on average somewhat younger, scored higher on the MCS and had a larger proportion of patients with $\geq 12$ weeks of postoperative PT compared to the excluded patients (SUPPLEMENTARY TABLE 1). 
Table 1

Characteristics of patients participating in a study on the use of physical therapy

\begin{tabular}{|c|c|c|c|c|c|c|c|c|c|}
\hline & THA & TKA & THA & & & & TKA & & \\
\hline & $n=1289$ & $\begin{array}{l}n= \\
1333\end{array}$ & $\begin{array}{l}\text { No } \\
\text { postoperative } \\
\text { PT }\end{array}$ & $\begin{array}{l}<12 \text { weeks } \\
\text { postoperative } \\
\text { PT }\end{array}$ & $\begin{array}{l}\geq 12 \text { weeks } \\
\text { postoperative } \\
\text { PT }\end{array}$ & $\begin{array}{l}\text { Difference } \\
\text { between } \\
\text { PT groups }\end{array}$ & $\begin{array}{l}\text { No } \\
\text { postoperative } \\
\text { PT }\end{array}$ & $\begin{array}{l}<12 \text { weeks } \\
\text { postoperative } \\
\text { PT }\end{array}$ & $\begin{array}{l}\geq 12 \text { weeks } \\
\text { postoperative } \\
\text { PT }\end{array}$ \\
\hline & & & $n=110$ & $n=503$ & $n=676$ & P Value & $\mathrm{n}=31$ & n = 377 & $n=925$ \\
\hline $\begin{array}{l}\text { Sex, female; n } \\
(\%)\end{array}$ & $\begin{array}{l}771 \\
(59.8)\end{array}$ & $\begin{array}{l}844 \\
(63.3)\end{array}$ & $63(57.3)$ & 279 (55.5) & 429 (63.5) & 0.018 & $7(54.8)$ & 218 (57.8) & 609 (65.8) \\
\hline Age, years & $68.3(8.8)$ & $\begin{array}{l}67.5 \\
(8.4)\end{array}$ & 69.9 (8.1) & $69.3(8.5)$ & $67.3(9.1)$ & $<0.001$ & $67.1(9.6)$ & 69.9 (7.9) & $66.5(8.4)$ \\
\hline $\begin{array}{l}\text { Body mass } \\
\text { index; } \mathrm{kg} / \mathrm{m}^{2}\end{array}$ & $27.2(4.2)$ & $\begin{array}{l}29.3 \\
(4.6)\end{array}$ & $27.4(4.9)$ & $27.1(4.1)$ & $27.4(4.2)$ & 0.409 & $28.9(5.4)$ & $29.6(4.7)$ & $29.2(4.5)$ \\
\hline $\begin{array}{l}\text { Living status; } \mathrm{n} \\
(\%)\end{array}$ & $\begin{array}{l}281 \\
(21.8)\end{array}$ & $\begin{array}{l}309 \\
(232)\end{array}$ & $36(32.7)$ & 104 (20.7) & 141 (20.9) & 0.015 & $12(38.7)$ & $100(26.5)$ & 197 (21.3) \\
\hline -With others & $\begin{array}{l}1008 \\
(78.2)\end{array}$ & $\begin{array}{l}1024 \\
(76.8)\end{array}$ & $74(67.3)$ & 399 (79.3) & 535 (79.1) & & 19 (61.3) & 277 (73.5) & 728 (78.7) \\
\hline $\begin{array}{l}\text { Working, yes; n } \\
(\%)\end{array}$ & $\begin{array}{l}306 \\
(23.9)\end{array}$ & $\begin{array}{l}327 \\
(24.5)\end{array}$ & $23(20.9)$ & 103 (20.6) & $180(26.8)$ & 0.036 & $5(16.1)$ & 57 (15.2) & 265 (29.0) \\
\hline $\begin{array}{l}\text { Working <67 } \\
\text { years }\end{array}$ & $\begin{array}{l}280 \\
(55.0)\end{array}$ & $\begin{array}{l}303 \\
(53.0)\end{array}$ & $21(56.8)$ & 91 (51.1) & 168 (57.3) & 0.412 & 5 (41.7) & 49 (42.6) & 249 (56.2) \\
\hline Comorbidity, & 501 & 557 & 47 (42.7) & 195 (38.8) & 259 (38.3) & 0.978 & 13 (41.9) & 159 (42.2) & 385 (41.6) \\
\hline$y(0,11(10)$ & & $(41.0)$ & 71 (64.5) & 291 (57.9) & 433 (64.1) & 0.381 & $20(64.5)$ & $238(63.1)$ & $536(60.39)$ \\
\hline Musculoskeletal & $(61.7)$ & $(61.6)$ & $3(2.7)$ & $21(4.2)$ & $41(6.1)$ & 0.176 & 4 (12.9) & $23(6.1)$ & $46(5.0)$ \\
\hline $\begin{array}{l}\text {-Non- } \\
\text { musculoskeletal }\end{array}$ & $65(5.0)$ & $\begin{array}{l}73 \\
(5.5)\end{array}$ & & & & & & & \\
\hline $\begin{array}{l}\text {-Sensory } \\
\text { impairments }\end{array}$ & & & & & & & & & \\
\hline $\begin{array}{l}\text { Comorbidity, } \\
\text { count, n (\%) }\end{array}$ & $\begin{array}{l}214 \\
(16.6)\end{array}$ & $\begin{array}{l}184 \\
(13.8)\end{array}$ & $14(12.7)$ & 88 (17.5) & 112 (16.6) & 0.45 & $5(16.1)$ & 43 (11.4) & 136 (14.7) \\
\hline 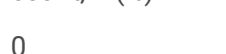 & & & 74 (67.3) & $294(58.4)$ & 370 (54.7) & & $16(51.6)$ & 207 (54.9) & 504 (54.5) \\
\hline 1 or 2 & (57.3) & (54.5) & $18(16.4)$ & 97 (19.3) & 165 (24.4) & & $8(25.8)$ & 97 (25.7) & $229(24.8)$ \\
\hline 3 or 4 & $\begin{array}{l}280 \\
(21.7)\end{array}$ & $\begin{array}{l}334 \\
(25.1)\end{array}$ & $4(3.6)$ & $24(4.8)$ & $29(4.3)$ & & $2(6.5)$ & $30(8.0)$ & $56(6.1)$ \\
\hline 5 or more & $57(4.4)$ & $\begin{array}{l}88 \\
(6.6)\end{array}$ & & & & & & & \\
\hline $\begin{array}{l}\text { Preoperative PT, } \\
\text { n (\%) }\end{array}$ & $\begin{array}{l}728 \\
(56.5)\end{array}$ & $\begin{array}{l}676 \\
(50.7)\end{array}$ & 63 (57.3) & 246 (50.9) & 419 (64.3) & $<0.001$ & 13 (41.9) & 167 (47.7) & 496 (55.6) \\
\hline Postoperative & 1038 & 1171 & - & $437(88.8)$ & 601 (90.2) & 0.433 & - & 331 (89.7) & 840 (93.3) \\
\hline $\begin{array}{l}\text {-Primary care } \\
\text {-Secondary care }\end{array}$ & $\begin{array}{l}120 \\
(10.4)\end{array}$ & $\begin{array}{l}98 \\
(7.7)\end{array}$ & & 55 (11.2) & 65 (9.8) & & & 38 (10.3) & $60(6.7)$ \\
\hline $\begin{array}{l}\text { Frequency } \\
\text { postoperative }\end{array}$ & $\begin{array}{l}556 \\
(475)\end{array}$ & $\begin{array}{l}397 \\
(306)\end{array}$ & - & 295 (59.4) & 261 (38.8) & $<0.001$ & - & 178 (47.5) & 219 (23.7) \\
\hline & $572(48.9)$ & 853 & & 178 (35.8) & 394 (58.5) & & & $190(50.7)$ & 663 (71.8) \\
\hline$-1 \times$ per week & $42(3.6)$ & (65.7) & & $24(4.8)$ & $18(2.7)$ & & & $7(1.9)$ & $41(4.4)$ \\
\hline $\begin{array}{l}-2 \times \text { per week } \\
-\geq 3 \times \text { per week }\end{array}$ & & $\begin{array}{l}48 \\
(3.7)\end{array}$ & & & & & & & \\
\hline
\end{tabular}

Legend to Table 1: Data is presented as mean and standard deviation between parentheses or reported otherwise.

HOOS= Hip disability and Osteoarthritis Outcome Score; KOOS= Knee injury and Osteoarthritis Outcome Score; NRS= Numeric Rating Scale; SF-12 = Short Fo THA=total hip arthroplasty; TKA= total knee arthroplasty; PT= Physical therapy 


\begin{tabular}{|c|c|c|c|c|c|c|c|c|c|}
\hline & THA & TKA & THA & & & & TKA & & \\
\hline HOOS or KOOS & 40.9 & 49.8 & $42.6(19.7)$ & $42.3(18.4)$ & $39.6(18.3)$ & 0.030 & $50.7(20.2)$ & $50.9(18.3)$ & $49.3(18.3)$ \\
\hline - Symptoms & & & $41.6(22.7)$ & 40.7 (18.5) & $37.6(17.7)$ & 0.008 & 37.1 (16.5) & $40.3(16.5)$ & 39.1 (17.8) \\
\hline - Pain & $(18.5)$ & $(17.4)$ & $43.1(21.5)$ & $43.4(19.0)$ & 39.9 (18.8) & 0.007 & $44.8(21.6)$ & 46.8 (17.7) & $45.6(18.2)$ \\
\hline$-A D L$ & $\begin{array}{l}41.6 \\
(19.2)\end{array}$ & $\begin{array}{l}45.9 \\
(18.1)\end{array}$ & $20.4(18.7)$ & 20.5 (19.1) & $18.7(18.8)$ & 0.236 & $10.5(16.5)$ & 11.5 (14.4) & $10.4(14.1)$ \\
\hline & $\begin{array}{l}19.6 \\
(18.9)\end{array}$ & $\begin{array}{l}10.7 \\
(14.2)\end{array}$ & & & & & & & \\
\hline & $\begin{array}{l}29.5 \\
(16.4)\end{array}$ & $\begin{array}{l}26.5 \\
(15.3)\end{array}$ & & & & & & & \\
\hline - Sport & & & $29.6(16.8)$ & $31.3(17.3)$ & $28.2(15.5)$ & 0.007 & $25.3(17.0)$ & 27.6 (15.5) & 26.1 (15.1) \\
\hline - Quality of life & & & & & & & & & \\
\hline NRS pain score; & $4.6(2.5)$ & 4.7 & $4.0(2.5)$ & $4.7(2.4)$ & $4.8(2.6)$ & 0.014 & $5.8(2.9)$ & $4.3(2.5)$ & $4.8(2.6)$ \\
\hline $\begin{array}{l}\text { - During rest } \\
\text { - During activity }\end{array}$ & $6.8(2.2)$ & $\begin{array}{l}6.7 \\
(2.5)\end{array}$ & $6.5(2.3)$ & $6.7(2.3)$ & $6.9(2.1)$ & 0.287 & $7.4(2.5)$ & $6.4(2.7)$ & $6.7(2.4)$ \\
\hline SF-12 & $\begin{array}{l}53.7 \\
(10.2)\end{array}$ & $\begin{array}{l}54.9 \\
(9.6)\end{array}$ & $53.4(11.7)$ & $54.1(9.8)$ & $53.4(10.3)$ & 0.434 & $53.6(10.7)$ & 55.1 (9.6) & 54.8 (9.6) \\
\hline $\begin{array}{l}\text { - MCS } \\
\text { - PCS }\end{array}$ & $32.9(6.2)$ & $\begin{array}{l}32.9 \\
(6.1)\end{array}$ & $32.5(6.5)$ & $33.2(6.3)$ & $32.8(6.0)$ & 0.333 & $33.3(6.1)$ & $32.9(6.1)$ & $32.8(6.1)$ \\
\hline $\begin{array}{l}\text { Duration of PT; } \\
\mathrm{n}(\%)\end{array}$ & $110(9.1)$ & $\begin{array}{l}31 \\
(2.2)\end{array}$ & & & & & & & \\
\hline - No PT & $(10.4)$ & $\begin{array}{l}75 \\
(5.3)\end{array}$ & & & & & & & \\
\hline-6 weeks- & $\begin{array}{l}160 \\
(13.2)\end{array}$ & $\begin{array}{l}142 \\
(10.1)\end{array}$ & & & & & & & \\
\hline - 8 weeks & $\begin{array}{l}217 \\
(17.9)\end{array}$ & $\begin{array}{l}160 \\
(11.3)\end{array}$ & & & & & & & \\
\hline ->12 week & $\begin{array}{l}676 \\
(55.9)\end{array}$ & $\begin{array}{l}925 \\
(65.5)\end{array}$ & & & & & & & \\
\hline
\end{tabular}

Legend to Table 1: Data is presented as mean and standard deviation between parentheses or reported otherwise.

HOOS= Hip disability and Osteoarthritis Outcome Score; KOOS= Knee injury and Osteoarthritis Outcome Score; NRS= Numeric Rating Scale; SF-12 = Short Fo THA=total hip arthroplasty; TKA= total knee arthroplasty; PT= Physical therapy

\section{Characteristics of THA and TKA patients}

Table 1 shows the baseline characteristics of the patients. Both THA/TKA patients were on average 68 years old (THA SD 8.8; and TKA SD 8.4 years), and 60\% and $63 \%$ of THA/TKA patients were female. Among patients younger than 67 years old, $55 \%$ and $53 \%$ of THA/TKA patients had a paid job before surgery. Overall, $39 \%$ of the THA patients had a musculoskeletal comorbidity, $62 \%$ had a non-musculoskeletal comorbidity and $5 \%$ had a sensory impairment. For the TKA group these numbers were respectively $42 \%, 62 \%$ and $6 \%$.

\section{Use of preoperative and postoperative PT}

Table 1 shows that 728 (57\%) THA patients and 676 (51\%) of the TKA patients received preoperative PT. After surgery 91\% of THA patients and 98\% of TKA received PT. The large majority used primary care PT and 676 (55.9\%) THA patients and 925 (65.5\%) TKA patients used it for 12 weeks or more, whereas $49 \%$ of THA patients and $66 \%$ of TKA patients reported an average frequency of PT of twice a week

Regarding the comparison of the characteristics of patients who had no postoperative PT or who had $<12$ weeks PT versus those who had $\geq 12$ weeks postoperative PT, it appeared that in both THA/TKA, patients with $\geq 12$ weeks postoperative PT were more often female, younger and employed. Also, they used preoperative PT more often and received $\geq 2$ sessions of postoperative PT treatments per week than patients who received PT $<12$ weeks. Additionally, the THA group with $\geq 12$ weeks postoperative PT scored lower on all HOOS subdomains apart from subdomain sport at baseline compared to the THA group with PT $<12$ weeks. The TKA group with $\geq 12$ weeks postoperative PT received PT in primary care more often. No differences on KOOS subdomains were found between both groups.

\section{Physical recovery and postoperative PT}

Table 2 shows the crude absolute HOOS/KOOS domain scores at 6 months as well as their changes compared to the preoperative scores for patients who received $<12$ weeks postoperative PT and those who received $\geq 12$ weeks postoperative PT. We found that the group with $\geq 12$ weeks postoperative PT had smaller improvements in HOOS/KOOS domain scores at 6 months. All differences between the groups were clinically relevant. 
Table 2

Physical recovery and postoperative physical therapy after total hip or knee arthroplasty surgery

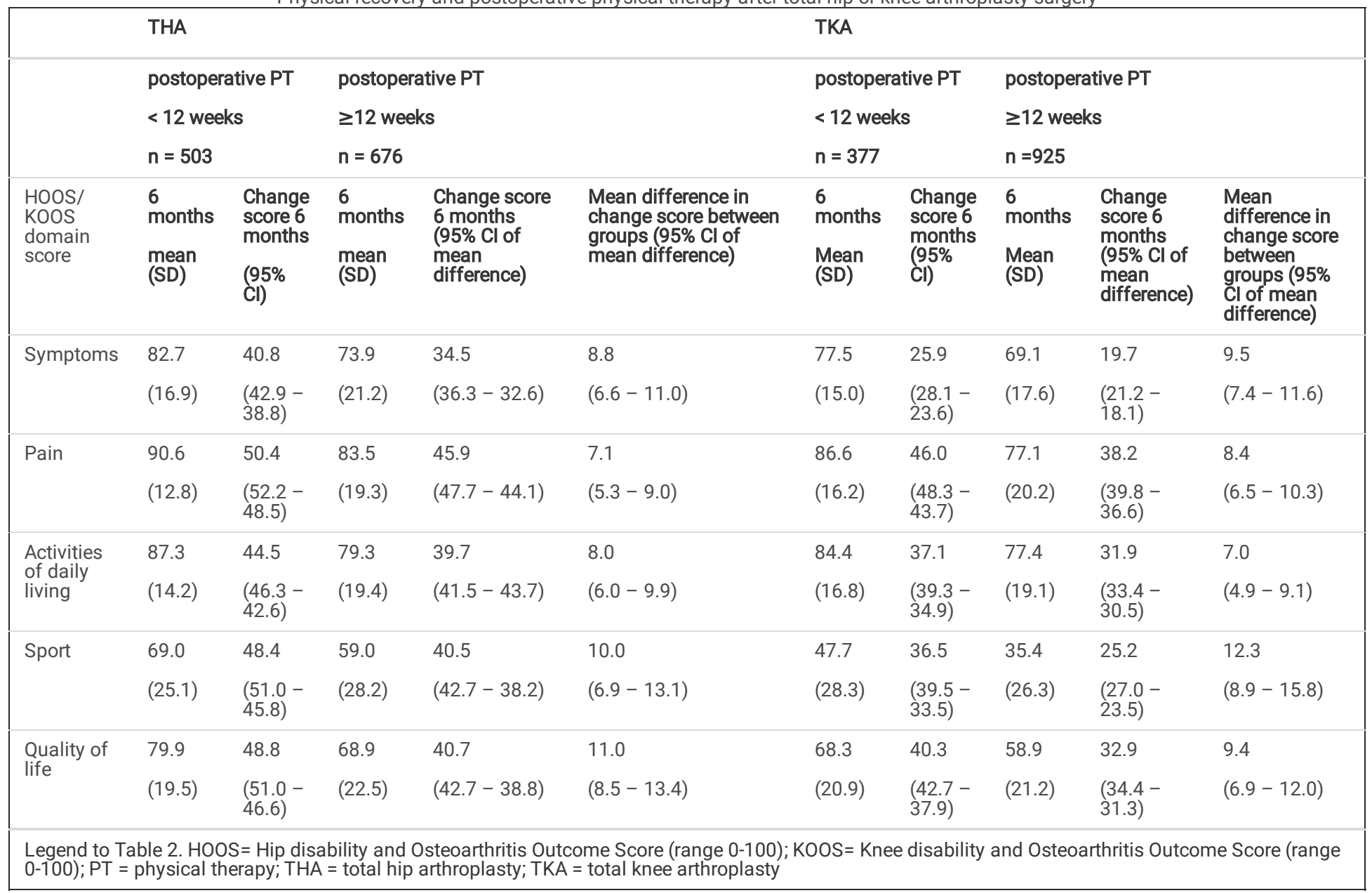

\section{Associations of recovery, comorbidities and duration of postoperative PT}

Tables 3 and 4 show the adjusted Odds Ratios of the associations between recovery on all subdomains of the HOOS/KOOS and the duration of postoperative PT including the influence of comorbidities within these associations. For both THA/TKA we found that better recovery at 6 months was associated with shorter postoperative PT on all subdomains of the HOOS/KOOS (Tables 3 and 4, model 1). 
Table 3

Association of duration of postoperative physical therapy and recovery in physical function in total hip arthroplasty patients 6 months after surgery

\begin{tabular}{|c|c|c|c|c|}
\hline & $\begin{array}{l}\text { Model } 1 \\
\text { Multivariable } \\
\text { OR [ } 95 \% \mathrm{Cl}]\end{array}$ & $\begin{array}{l}\text { Model } 1+\text { Musculoskeletal } \\
\text { comorbidities } \\
\text { OR [ } 95 \% \mathrm{Cl}]\end{array}$ & $\begin{array}{l}\text { Model } 1+\text { Non-musculoskeletal } \\
\text { comorbidities } \\
\text { OR [ } 95 \% \mathrm{Cl}]\end{array}$ & $\begin{array}{l}\text { Model } 1+\text { Sensory } \\
\text { Impairments } \\
\text { OR [ } 95 \% \mathrm{Cl}]\end{array}$ \\
\hline HOOS ADL & \multirow{3}{*}{$\begin{array}{l}0.97[0.96- \\
0.98] \\
\text { NA } \\
\text { NA }\end{array}$} & $0.96[0.95-0.98]$ & $0.95[0.93-0.97]$ & $0.97[0.96-0.98]$ \\
\hline Comorbidity & & $0.15[0.03-0.87]$ & $0.71[0.01-0.59]$ & $0.23[0.03-2.20]$ \\
\hline ADL*comorbidity & & $1.02[1.00-1.04]$ & $1.03[1.01-1.06]$ & $1.02[0.99-1.05]$ \\
\hline HOOS Pain & \multirow{3}{*}{$\begin{array}{l}0.97[0.97- \\
0.98] \\
\text { NA } \\
\text { NA }\end{array}$} & $0.97[0.96-0.99]$ & $0.94[0.91-0.96]$ & $0.97[0.96-0.98]$ \\
\hline Comorbidity & & $0.62[0.10-3.75]$ & $0.01[0.001-0.17]$ & $0.23[0.02-2.71]$ \\
\hline Pain*comorbidity & & $1.01[0.99-1.03]$ & $1.05[1.02-1.08]$ & $1.02[0.99-1.05]$ \\
\hline HOOS Symptoms & \multirow{3}{*}{$\begin{array}{l}0.98[0.97- \\
0.98] \\
\text { NA } \\
\text { NA }\end{array}$} & $0.97[0.96-0.99]$ & $0.96[0.94-0.97]$ & $0.98[0.97-0.98]$ \\
\hline Comorbidity & & $0.39[0.10-1.45]$ & $0.19[0.04-0.82]$ & $0.52[0.08-3.34]$ \\
\hline Symptoms*comorbidity & & $1.01[0.96-1.03]$ & $1.03[1.0-1.04]$ & $1.01[0.99-1.04]$ \\
\hline HOOS Sport and Recreation & \multirow{3}{*}{$\begin{array}{l}0.99[0.98- \\
0.99]^{[} \\
\text {NA } \\
\text { NA }\end{array}$} & $0.98[0.97-0.99]$ & $0.98[0.97-0.99]$ & $0.99[0.98-0.99]$ \\
\hline Comorbidity & & $0.39[0.17-0.85]$ & $0.74[0.32-1.67]$ & $0.52[0.16-1.67]$ \\
\hline $\begin{array}{l}\text { Sport and } \\
\text { Recreation*comorbidity }\end{array}$ & & $1.02[1.00-1.03]$ & $1.01[1.00-1.02]$ & $1.02[1.00-1.04]$ \\
\hline HOOS QoL & \multirow{3}{*}{$\begin{array}{l}0.98[0.97- \\
0.98] \\
\text { NA } \\
\text { NA }\end{array}$} & $0.97[0.96-0.99]$ & $0.97[0.95-0.98$ & $0.98[0.97-0.98]$ \\
\hline Comorbidity & & $0.64[0.20-1.99]$ & $0.99[0.99-1.00]$ & $0.51[0.09-3.06]$ \\
\hline QoL*comorbidity & & $1.01[0.99-1.02]$ & $1.02[1.00-1.03]$ & $1.01[0.99-1.04]$ \\
\hline \multicolumn{5}{|c|}{ Legend to Table 3. ADL= Activities of daily living; HOOS= Hip disability and Osteoarthritis Outcome Score; THA = total hip arthroplasty } \\
\hline \multicolumn{5}{|c|}{$\begin{array}{l}\text { Model 1: long duration of PT = bo+b1x (HOOS score) adjusted for HOOS score baseline, BMI, age, sex, living status, and Short Form-12 Mental Componen } \\
\text { Summary scale and HOOS score baseline. }\end{array}$} \\
\hline
\end{tabular}


Table 4

Association of recovery and duration of postoperative physical therapy in total knee arthroplasty patients 6 months after surgery

\begin{tabular}{|c|c|c|c|c|}
\hline & $\begin{array}{l}\text { Model } 1 \\
\text { Multivariable } \\
\text { OR [ } 95 \% \mathrm{Cl}]\end{array}$ & $\begin{array}{l}\text { Model } 1+\text { Musculoskeletal } \\
\text { comorbidities } \\
\text { OR [ } 95 \% \mathrm{Cl}]\end{array}$ & $\begin{array}{l}\text { Model } 1+\text { Non-musculoskeletal } \\
\text { comorbidities } \\
\text { OR [ } 95 \% \mathrm{Cl}]\end{array}$ & $\begin{array}{l}\text { Model } 1+\text { Sensory } \\
\text { Impairments } \\
\text { OR [ } 95 \% \mathrm{Cl}]\end{array}$ \\
\hline KOOS ADL & \multirow{3}{*}{$\begin{array}{l}0.97[0.97- \\
0.98] \\
\text { NA } \\
\text { NA }\end{array}$} & $0.99[0.98-1.00]$ & $0.98[0.96-1.00]$ & $0.97[0.96-0.98]$ \\
\hline Comorbidity & & $5.78[1.21-27.67]$ & $1.64[0.24-11.04]$ & $1.41[0.12-17.14]$ \\
\hline ADL*comorbidity & & $0.98[0.96-1.00]$ & $0.99[0.97-1.01]$ & $0.99[0.96-1.03]$ \\
\hline KOOS Pain & \multirow{3}{*}{$\begin{array}{l}0.97[0.96- \\
0.98] \\
\text { NA } \\
\text { NA }\end{array}$} & $0.99[0.97-1.00]$ & $0.98[0.97-1.00]$ & $0.97[0.96-0.98]$ \\
\hline Comorbidity & & $6.17[1.23-30.92]$ & $3.95[0.66-23.60]$ & $0.83[0.06-2.32]$ \\
\hline Pain*comorbidity & & $0.98[0.96-1.00]$ & $0.98[0.96-1.00]$ & $1.00[0.97-1.03]$ \\
\hline KOOS Symptoms & \multirow{3}{*}{$\begin{array}{l}0.97[0.96- \\
0.98] \\
\text { NA } \\
\text { NA }\end{array}$} & $0.98[0.97-1.00]$ & $0.97[0.95-0.99]$ & $0.97[0.96-0.98]$ \\
\hline Comorbidity & & $3.55[0.76-16.60]$ & $0.93[0.18-4.91]$ & $5.56[0.24-131.75]$ \\
\hline Symptoms*comorbidity & & $0.99[0.97-1.01]$ & $1.00[0.98-1.02]$ & $0.97[0.93-1.02]$ \\
\hline KOOS Sport and Recreation & \multirow{3}{*}{$\begin{array}{l}0.98[0.98- \\
0.99]^{0} \\
\text { NA } \\
\text { NA }\end{array}$} & $0.99[0.98-0.99]$ & $0.98[0.97-0.99]$ & $0.98[0.98-0.99]$ \\
\hline Comorbidity & & $0.98[0.53-1.82]$ & $0.92[0.47-1.78]$ & $1.24[0.45-3.42]$ \\
\hline $\begin{array}{l}\text { Sport and } \\
\text { Recreation*comorbidity }\end{array}$ & & $1.00[0.99-1.01]$ & $1.00[0.99-1.01]$ & $0.98[0.96-1.01]$ \\
\hline KOOS QoL & \multirow{3}{*}{$\begin{array}{l}0.98[0.97- \\
0.98] \\
\text { NA } \\
\text { NA }\end{array}$} & $0.99[0.98-1.00]$ & $0.98[0.97-0.99]$ & $0.98[0.97-0.99]$ \\
\hline Comorbidity & & $2.27[0.82-6.29]$ & $0.90[0.29-02.80]$ & $1.05[0.23-4.76]$ \\
\hline QoL*comorbidity & & $0.99[0.97-1.00]$ & $1.00[0.98-1.02]$ & $1.00[0.97-1.02]$ \\
\hline
\end{tabular}

Legend to Table 4. ADL= Activities of daily living; KOOS= Knee disability and Osteoarthritis Outcome Score; TKA = total knee arthroplasty

Model 1: long duration of PT=bo+b1x (KOOS score) adjusted for KOOS score baseline, BMI, age, sex, living status, Short Form-12 Mental Component Summary scale and KOOS score baseline.

In the other models comorbidities (musculoskeletal, non-musculoskeletal or sensory impairment) and their interaction with recovery were also taken into account.

In THA patients, the existence of non-musculoskeletal comorbidities modified the associations between recovery and the duration of postoperative PT (Table

3). In patients with a non-musculoskeletal comorbidity, the impact of physical recovery on the risk of long term PT was smaller than in the patients without musculoskeletal comorbidity (SUPPLEMENTARY FIGURE 1-A). Hence in patients with a low HOOS subscore the probability of $>12$ weeks of PT therapy was lower in patients with a non-musculoskeletal as compared to patients without a non-musculoskeletal comorbidity. However, in patients with high HOOS subscores this probability was higher in patients with a non-musculoskeletal comorbidity than in patients without such a comorbidity. Moreover, the presence of a musculoskeletal comorbidity was identified as an effect modifier with regard to Activities of daily living (ADL) and Sport and recreation recovery and postoperative PT. Sensory comorbidities only had an effect on Sport and recreation recovery and postoperative PT. In TKA patients (Table 4) the existence of comorbidities did not modify the associations between recovery and the duration of postoperative PT (Table 4).

\section{Discussion}

We evaluated whether the patient's recovery 6 months after THA/TKA, including the presence of comorbidities, was related to the duration of postoperative PT. Approximately $95 \%$ of the study population received postoperative PT in a primary setting, where $49 \%$ of THA patients and $66 \%$ of TKA patients reported an average frequency of PT of twice a week. In about $56 \%$ of the THA and $66 \%$ of the TKA patients the average duration of postoperative PT was 12 weeks or more. Worse recovery at 6 months was associated with a longer duration of postoperative PT. The association between physical recovery and the use of longterm postoperative PT was weaker in THA patients with comorbidities than in patients without such a comorbidity. In TKA patients, comorbidities did not modify the association between recovery and postoperative PT duration.

Compared to Peter et al.(10), the frequency and duration of postoperative PT was slightly lower, but it was still substantially high compared to other Western countries(10-12). Our findings regarding the patient's recovery and duration of postoperative PT are in line with the study of Smith et al(12). They found that THA/TKA patients who received more than 10 postoperative PT sessions reported more complaints on average one year after surgery than patients who had less PT sessions. However, in the study of Smith et al., no adjustments were made and comorbidities were not taken into account. Nevertheless, the finding of both previous as well as the current study suggest that patients who were likely to have a greater need of PT due to insufficient recovery, indeed received it over a prolonged period. Hence, although currently clear evidence-based postoperative PT treatment protocols are absent, physical therapists seem to be able to adapt the PT treatment duration to the individual patient needs. 
In $85 \%$ of our population at least one comorbidity was present, which is similar to previously published prevalence's in OA patients, which ranged from $68 \%-85 \%(36-39)$. In THA patients, the presence of musculoskeletal, non-musculoskeletal or sensory impairments modified the association between recovery and postoperative PT in at least one of the HOOS domains. In THA patients with such comorbidities the effect of recovery on the use of long-term postoperative PT was smaller than patients without such comorbidities. This may be explained by the impact a comorbidity has on the postoperative PT treatment. Hence, the presence of comorbidities may require a more tailored PT treatment approach, in which the physical complaints resulting from the comorbidity can have a more prominent role and the recovery of the THA surgery may be less leading(40). For example, a patient with heart failure may not be able to perform strenuous exercise, thereby some complaints possibly cannot be improved as much as when these exercises could be performed. Moreover, for these patients improvement of the aerobic capacity may be an additional goal. As such, the PT goals will differ from patients without such a comorbidity. In TKA patients, the presence of comorbidities had no moderating effect on the effect of recovery and long-term PT. A possible explanation could be that recovery after TKA is more complex and takes a longer period of time than after THA(41), as such the role of a comorbidity on the PT treatment may be less pronounced.

Nonetheless, these protocols could be important in the delivery of the most optimal composition, dosage and mode of PT treatment(42). Hence, the rising number of arthroplasty surgeries and their accompanying strain on the health care systems and society as a whole warrant for a very efficient postoperative PT approach. Here there may be a role for a multidisciplinary consultation between health care providers (general practitioners, orthopedic surgeons, physical therapists), thereby improving communication, and increasing knowledge and agreement on rehabilitation treatment. Additionally, monitoring the recovery of a patient by a healthcare provider appears to be beneficial for better outcomes (43). Future studies should focus on the possibilities to improve or accelerate recovery using PT, thereby decreasing the duration of PT for THA/TKA patients. The PT has an excellent position to monitor the recovery process and identify patients with insufficient improvement due to the fact that the PT sees a patient twice a week on average in the first weeks after surgery, as opposed to a regular consultation by the orthopedic surgeon which is usually scheduled once in this period.

A strength of this study is the large regional, multicenter prospective cohort study design, in which we obtained both preoperative and postoperative assessments. Secondly, we used validated surveys to obtain recovery scores after surgery. However, this could also be a possible limitation, as we asked patients at six months about the duration of PT, which could have induced some recall bias. Examination of medical records could have provided more reliable and additional information, such as the content of the PT treatment. However, this method could not be used in the framework of this study. Another possible limitation could be generalizability; all patients were included in the Netherlands, therefore the results may not be one to one generalizable to other countries with different health insurance systems. Nevertheless, the sociodemographic and clinical characteristics of the participants were quite similar to those of persons who underwent THA/TKA in other observational studies(16, 27, 39, 44, 45).

\section{Conclusion}

In conclusion, slower recovery was associated with longer duration of postoperative PT use after THA/TKA. The impact of physical recovery on the use of long-term postoperative PT was smaller in THA patients with comorbidities, but not in TKA patients. Patients with longer postoperative PT use showed worse recovery than patients with a shorter duration of PT use, suggesting that PT provision is in line with patients' needs.

\section{List Of Abbreviations}

LOAS: Leiden Orthopaedics Outcomes of Osteoarthritis Study

PT: Physical therapy

THA: Total hip arthroplasty

TKA: Total knee arthroplasty

HOOS: The Hip disability and Osteoarthritis Outcome Score

KOOS: Knee injury and Osteoarthritis Outcome Score

OA: osteoarthritis

BMI: Body Mass Index

NRS: Numeric Rating Scale

SF-12: Short Form-12

PCS: Physical Component Summary scale

MCS: Mental Component Summary scale

CBS: Dutch Central Bureau of Statistics

MCID: Minimal Clinical Important Differences

SD: standard deviation 
Cl: confidence interval

ADL: Activities of daily living

\section{Declarations}

\section{Ethics approval and consent to participate}

Ethical approval was obtained from the Medical Ethics Committee of the Leiden University Medical Center (Registration number P12.047). All participants signed an informed consent form prior to start of the study.

\section{Consent for publication}

No individual person's data will be published, therefore consent for publication is not applicable.

\section{Availability of data and materials}

Data is owned by a third party. The data underlying this article were provided by the LOAS study group by permission. The data will be shared on reasonable request to the corresponding author, with permission of the LOAS study group.

\section{Competing interests}

None of the authors have a conflicts of interest to disclose.

\section{Funding}

This study was funded by the Dutch Arthritis Society and the Department of Orthopedics of the Leiden University Medical Center, Leiden, the Netherlands.

\section{Authors contribution}

LG,DL,TVV and MG have contributed to the conception of this study. LG, DL,MG did the analysis and wrote this manuscript. All authors were involved in revising the manuscript critically for important intellectual content. All authors read and approved the final manuscript. All authors agree to be accountable for all aspects of the work in ensuring that questions related to the accuracy or integrity of any part of the work are appropriately investigated and resolved.

\section{Acknowledgements}

Not Applicable.

\section{References}

1. LROI. Online LROI-report 20182018 [Available from: https://www.Iroi-report.nl/previous-reports/online-Iroi-report-2018/.

2. OECD. Health at a Glance 2017: OECD Indicators, OECD Publishing; 2017 [Available from: https://www.oecd-ilibrary.org/social-issues-migrationhealth/health-at-a-glance-2017_health_glance-2017-en;jsessionid=sHVPNloegsvfCp0tRJdc6BR7.ip-10-240-5-110.

3. Lowe CJM, Barker KL, Dewey M, Sackley CM. Effectiveness of physiotherapy exercise after knee arthroplasty for osteoarthritis: systematic review and meta-analysis of randomised controlled trials. British Medical Journal. 2007;335(7624):812-5.

4. Minns Lowe CJ, Barker KL, Dewey ME, Sackley CM. Effectiveness of physiotherapy exercise following hip arthroplasty for osteoarthritis: a systematic review of clinical trials. BMC Musculoskelet Disord. 2009;10:98.

5. Rahmann AE, Brauer SG, Nitz JC. A specific inpatient aquatic physiotherapy program improves strength after total hip or knee replacement surgery: a randomized controlled trial. Arch Phys Med Rehabil. 2009;90(5):745-55.

6. Liebs TR, Herzberg W, Ruther W, Haasters J, Russlies M, Hassenpflug J. Ergometer cycling after hip or knee replacement surgery: a randomized controlled trial. J Bone Joint Surg Am. 2010;92(4):814-22.

7. Westby MD, Brittain A, Backman CL. Expert consensus on best practices for post-acute rehabilitation after total hip and knee arthroplasty: a Canada and United States Delphi study. Arthritis Care Res (Hoboken). 2014;66(3):411-23.

8. Westby MD, Marshall DA, Jones CA. Development of quality indicators for hip and knee arthroplasty rehabilitation. Osteoarthritis Cartilage. 2018;26(3):370-82.

9. Kampshoff dCS, Peter dWFH, MSc MCMvD, Knoop dJ, MSc GAM, Vlieland pdTPMV. KNGF-richtlijn Artrose heup-knie. Conservatieve, pre- en postoperatieve behandeling. 2018.

10. Peter WF, Tilbury C, Verdegaal SHM, Onstenk R, Vehmeijer SB, Vermeulen EM, et al. The provision of preoperative and postoperative physical therapy in elderly people with hip and knee osteoarthritis undergoing primary joint replacement surgery. Current Orthopaedic Practice. 2016;27:173-83.

11. Hamilton DF, Loth FC, MacDonald DJ, MacFarlane GJ, Beard DJ, Simpson AHR, et al. Exploring variation in patient access of post-discharge physiotherapy following total hip and knee arthroplasty under a choice based system in the UK: an observational cohort study. BMJ Open.

2019;9(2):e021614. 
12. Smith TO, Dainty JR, Clark EM, Whitehouse MR, Price AJ, MacGregor AJ. Demographic and geographical variability in physiotherapy provision following hip and knee replacement. An analysis from the National Joint Registry for England, Wales, Northern Ireland and the Isle of Man. Physiotherapy. 2020;106:1-11.

13. Hussain SM, Wang Y, Shaw JE, Wluka AE, Graves S, Gambhir M, et al. Relationship of weight and obesity with the risk of knee and hip arthroplasty for osteoarthritis across different levels of physical performance: a prospective cohort study. Scand J Rheumatol. 2019;48(1):64-71.

14. Wang Y, Wluka AE, Simpson JA, Giles GG, Graves SE, de Steiger RN, et al. Body weight at early and middle adulthood, weight gain and persistent overweight from early adulthood are predictors of the risk of total knee and hip replacement for osteoarthritis. Rheumatology (Oxford). 2013;52(6):103341.

15. Peter WF, Nelissen RG, Vlieland TP. Guideline recommendations for post-acute postoperative physiotherapy in total hip and knee arthroplasty: are they used in daily clinical practice? Musculoskeletal Care. 2014;12(3):125-31.

16. Artz N, Dixon S, Wylde V, Beswick A, Blom A, Gooberman-Hill R. Physiotherapy provision following discharge after total hip and total knee replacement: a survey of current practice at high-volume NHS hospitals in England and wales. Musculoskeletal Care. 2013;11(1):31-8.

17. Chen HW, Chen HM, Wang YC, Chen PY, Chien CW. Association between rehabilitation timing and major complications of total knee arthroplatsy. J Rehabil Med. 2012;44(7):588-92.

18. Wijnen A, Bouma SE, Seeber GH, van der Woude LHV, Bulstra SK, Lazovic D, et al. The therapeutic validity and effectiveness of physiotherapeutic exercise following total hip arthroplasty for osteoarthritis: A systematic review. PLoS One. 2018;13(3):e0194517.

19. Naylor JM, Hart A, Harris IA, Lewin AM. Variation in rehabilitation setting after uncomplicated total knee or hip arthroplasty: a call for evidence-based guidelines. BMC Musculoskelet Disord. 2019;20(1):214.

20. Benz T, Angst F, Oesch P, Hilfiker R, Lehmann S, Mueller Mebes C, et al. Comparison of patients in three different rehabilitation settings after knee or hip arthroplasty: a natural observational, prospective study. BMC Musculoskelet Disord. 2015;16:317.

21. Moutzouri M, Gleeson N, Billis E, Tsepis E, Gliatis J. Greek Physiotherapists' Perspectives on Rehabilitation Following Total Knee Replacement: a Descriptive Survey. Physiother Res Int. 2017;22(4)

22. Buirs LD, Van Beers LW, Scholtes VA, Pastoors T, Sprague S, Poolman RW. Predictors of physical functioning after total hip arthroplasty: a systematic review. BMJ Open. 2016;6(9):e010725.

23. Lungu E, Vendittoli PA, Desmeules F. Preoperative Determinants of Patient-reported Pain and Physical Function Levels Following Total Knee Arthroplasty: A Systematic Review. Open Orthop J. 2016;10:213-31.

24. Lungu E, Maftoon S, Vendittoli PA, Desmeules F. A systematic review of preoperative determinants of patient-reported pain and physical function up to 2 years following primary unilateral total hip arthroplasty. Orthop Traumatol Surg Res. 2016;102(3):397-403.

25. Heiberg KE, Bruun-Olsen V, Ekeland A, Mengshoel AM. Effect of a walking skill training program in patients who have undergone total hip arthroplasty: Followup one year after surgery. Arthritis Care Res (Hoboken). 2012;64(3):415-23.

26. van der Leeden M, Stuiver MM, Huijsmans R, Geleijn E, de Rooij M, Dekker J. Structured clinical reasoning for exercise prescription in patients with comorbidity. Disabil Rehabil. 2018:1-6.

27. Peter WF, Dekker J, Tilbury C, Tordoir RL, Verdegaal SH, Onstenk R, et al. The association between comorbidities and pain, physical function and quality of life following hip and knee arthroplasty. Rheumatol Int. 2015;35(7):1233-41.

28. Tilbury C, Leichtenberg CS, Kaptein BL, Koster LA, Verdegaal SHM, Onstenk R, et al. Feasibility of Collecting Multiple Patient-Reported Outcome Measures Alongside the Dutch Arthroplasty Register. Journal of Patient Experience. 2019;0(0):2374373519853166.

29. McCaffery M. Using the 0-to-10 pain rating scale. Am J Nurs. 2001;101(10):81-2.

30. Gandek B, Ware JE, Aaronson NK, Apolone G, Bjorner JB, Brazier JE, et al. Cross-validation of item selection and scoring for the SF-12 Health Survey in nine countries: results from the IQOLA Project. International Quality of Life Assessment. J Clin Epidemiol. 1998;51(11):1171-8.

31. Reeuwijk KG, de Rooij M, van Dijk GM, Veenhof C, Steultjens MP, Dekker J. Osteoarthritis of the hip or knee: which coexisting disorders are disabling? Clin Rheumatol. 2010;29(7):739-47.

32. CBS. Zelfgerapporteerde medische consumptie, gezondheid en leefstijl Den Haag/Heerlen2010 [Available from: http://www.scp.nl/Onderzoek/Bronnen/Beknopte_onderzoeksbeschrijvingen/Permanent_onderzoek_naar_de_leefsituatie_POLS.

33. de Groot IB, Reijman M, Terwee CB, Bierma-Zeinstra SM, Favejee M, Roos EM, et al. Validation of the Dutch version of the Hip disability and Osteoarthritis Outcome Score. Osteoarthritis Cartilage. 2007;15(1):104-9.

34. de Groot IB, Favejee MM, Reijman M, Verhaar JA, Terwee CB. The Dutch version of the Knee Injury and Osteoarthritis Outcome Score: a validation study. Health Qual Life Outcomes. 2008;6:16.

35. Lyman S, Lee YY, McLawhorn AS, Islam W, MacLean CH. What Are the Minimal and Substantial Improvements in the HOOS and KOOS and JR Versions After Total Joint Replacement? Clin Orthop Relat Res. 2018;476(12):2432-41.

36. Caporali R, Cimmino MA, Sarzi-Puttini P, Scarpa R, Parazzini F, Zaninelli A, et al. Comorbid conditions in the AMICA study patients: effects on the quality of life and drug prescriptions by general practitioners and specialists. Semin Arthritis Rheum. 2005;35(1 Suppl 1):31-7.

37. Tuominen U, Blom M, Hirvonen J, Seitsalo S, Lehto M, Paavolainen P, et al. The effect of co-morbidities on health-related quality of life in patients placed on the waiting list for total joint replacement. Health Qual Life Outcomes. 2007;5:16

38. van Dijk GM, Veenhof C, Schellevis F, Hulsmans H, Bakker JP, Arwert H, et al. Comorbidity, limitations in activities and pain in patients with osteoarthritis of the hip or knee. BMC Musculoskelet Disord. 2008;9:95. 
39. Meessen JM, Peter WF, Wolterbeek R, Cannegieter SC, Tilbury C, Benard MR, et al. Patients who underwent total hip or knee arthroplasty are more physically active than the general Dutch population. Rheumatol Int. 2017;37(2):219-27.

40. de Rooij M, van der Leeden M, van der Esch M, Lems WF, Meesters JJL, Peter WF, et al. Evaluation of an educational course for primary care physiotherapists on comorbidity-adapted exercise therapy in knee osteoarthritis: an observational study. Musculoskeletal Care. 2020;18(2):122-33.

41. Bourne RB, Chesworth B, Davis A, Mahomed N, Charron K. Comparing patient outcomes after THA and TKA: is there a difference? Clin Orthop Relat Res. 2010;468(2):542-6.

42. van Doormaal MCM, Meerhoff GA, Vliet Vlieland TPM, Peter WF. A clinical practice guideline for physical therapy in patients with hip or knee osteoarthritis. Musculoskeletal Care. 2020.

43. D LS, Hipango J, Sinnott KA, Dunn JA, Rothwell A, Hsieh CJ, et al. Rehabilitation after total joint replacement: a scoping study. Disabil Rehabil. 2018;40(14):1718-31.

44. Naylor J, Harmer A, Fransen M, Crosbie J, Innes L. Status of physiotherapy rehabilitation after total knee replacement in Australia. Physiother Res Int. 2006;11(1):35-47.

45. de Beer J, Petruccelli D, Adili A, Piccirillo L, Wismer D, Winemaker M. Patient perspective survey of total hip vs total knee arthroplasty surgery. J Arthroplasty. 2012;27(6):865-9 e1-5.

\section{Figures}

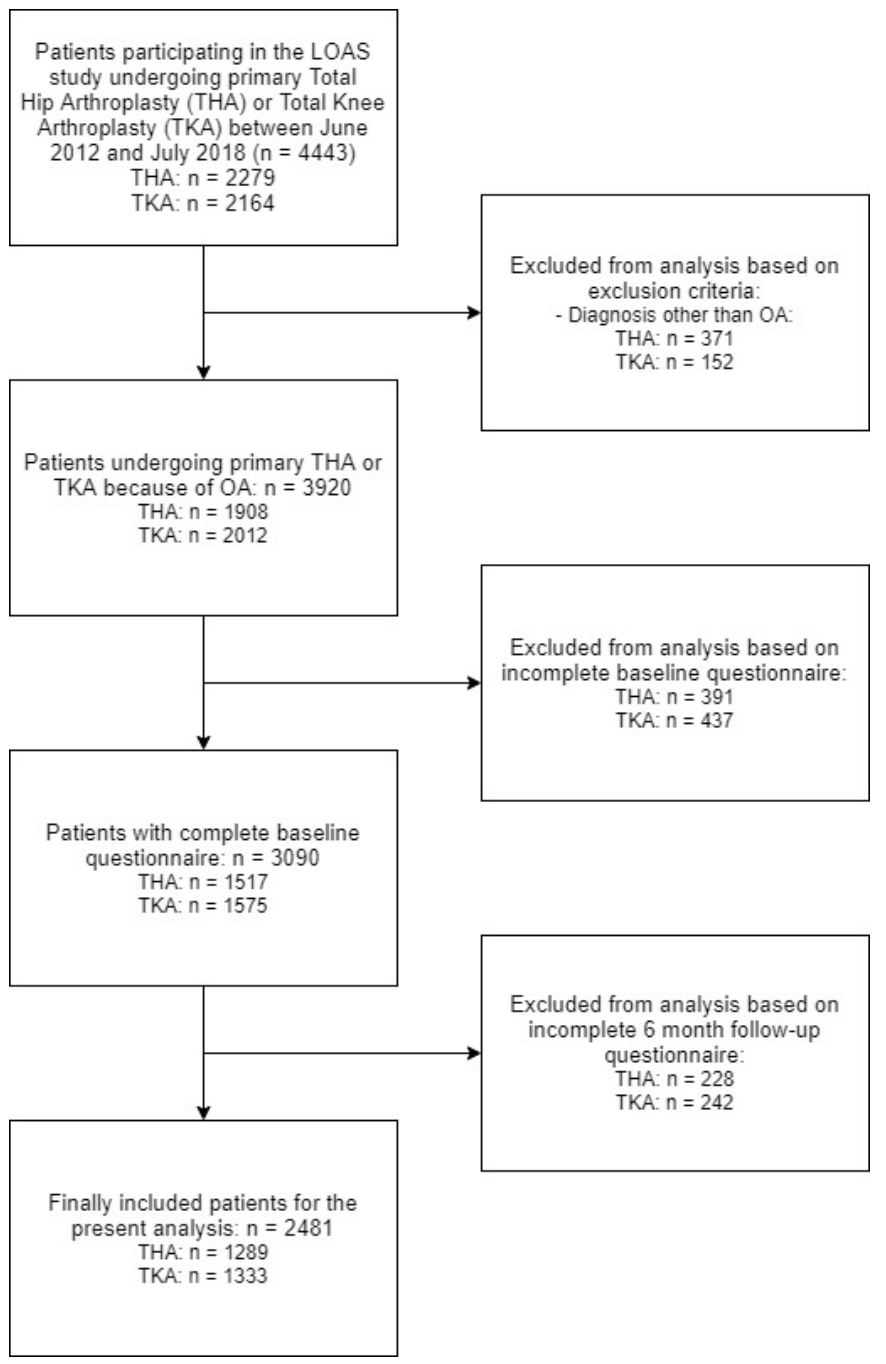

\section{Figure 1}

Flowchart of patients in a study on the use of physical therapy before and after THA/TKA

\section{Supplementary Files}

This is a list of supplementary files associated with this preprint. Click to download. 